\title{
Academic Delay of Gratification: A Construct Validation with High School Students
}

\begin{abstract}
This study utilized a construct validation approach to the empirical assessment of the Arabic version of the Academic Delay of Gratification Scale (ADOGS). A construct validation approach utilized both within-network and between-network constructs validation in two samples of Egyptian adolescents. For the within-network construct validation, confirmatory factor analysis (CFA) revealed that the single factor model of the ADOGS had adequate fit to the data of both sample, whereas the hierarchical, the correlated two-factor, the uncorrelated (orthogonal) two-factor models, and the threefactor model did not. Multigroup CFAs revealed full gender invariance for the ADOGS unidimensional model in both samples. A latent mean analysis showed consistent gender differences on the ADOGS in the females' direction when males and females were compared within both samples. The ADOGS correlated positively with liking, importance, expectancy, and utility but negatively with negative consequences, and time/effort for the delay versus non-delay alternatives in both samples. The ADOGS correlated positively with academic achievement in both samples but not with selfreported social desirability in either sample.
\end{abstract}

Keywords: Academic delay of gratification; Confirmatory factor analysis; Constructvalidation; Adolescents; Egypt

Research Article
Volume 8 Issue 1 - 2017
Sabry M Abd-El-Fattah ${ }^{1 *}$ and Alshaimaa M
Sallman ${ }^{2}$
${ }^{1}$ Department of Educational Psychology, Minia University,
Egypt
${ }^{2}$ Department of Mental Health, Minia University, Egypt
*Corresponding author: Sabry M Abd-El-Fattah,
Department of Educational Psychology, Faculty of Education,
Minia University, Minia City, Egypt,
Email: sabryrahma@ @hotmail.com
Received: July 02, 2017 | Published: August 24, 2017

\section{Introduction}

Beginning in the late-1960s and continuing into the early 1970s, Walter Mischel et al. [1,2] have conducted a series of studies, known as the Stanford marshmallow experiment, to measure children's willpower to defer gratification. In these experiments, a preschooler would be given two marshmallows if she waited until the researcher returned to the room after finishing a task and one marshmallow if she could not wait and rang a bell to call the researcher back to the room at any time. The length of time individual children waited until ringing the bell was taken as a measure of their ability to delay gratification. According to Mischel et al. [3-5], delay of gratification represents people's attempts to delay an attractive, immediately obtainable goal (e.g., get one marshmallow immediately) in order to pursue long-range objectives (e.g., wait for few minutes and get two marshmallows). Delay of gratification has been conceived as an ability or competence [5] that children develop over time and as a relatively stable generalized disposition [6]. Furthermore, various methods have been used to manipulate and measure delay of gratification with older populations including Q-sorts [7] that involve persons ordering a series of statements about themselves, and surveys. Survey items are designed to present choices that were representative of adults' experiences rather than the reward size options offered to young children in the Stanford marshmallow experiment [1,2]. For example, Ray \& Najman's [8] Deferment of Gratification Questionnaire (DGQ) assessed students' general disposition to delay gratification (e.g., "Are you good at saving your money rather than spending it straight away?") [9]. developed the Multidimensional Delay of
Gratification (MDG) scale that targeted broad academic-related issues (e.g., "Rent an expensive apartment, or save money for later after graduation").

\section{Academic delay of gratification}

From an academic perspective, many students strive to remain goal oriented and committed to tasks while facing distractions that are typical features of learning contexts. These distractions may include turning to more enjoyable activities such as going out to party with friends, doing shopping, and going to cinema or theatre. Because remaining goal oriented and committed to tasks often involve foregoing an attractive, immediately obtainable goal (e.g., going to a party) in order to pursue long-range academic objectives (e.g., obtain a high score on a test), this process can be linked to delay of gratification [1]. Bembenutty \& Karabenick [10] defined academic delay of gratification as 'students' postponement of immediately available opportunities to satisfy impulses in favor of pursuing chosen important academic rewards or goals that are temporally remote but ostensibly more valuable."

\section{The Academic Delay of Gratification Scale (ADOGS)}

Following the approach of Pintrich and his colleagues [11,12], Bembenutty \& Karabenick [13] developed the Academic Delay of Gratification Scale (ADOGS) to assess individuals' tendencies to delay gratification within specific academic situations rather than a general personality trait. In developing the ADOGS, Bembenutty \& Karabenick constructed an item pool of 16 items by borrowing some items from the MDG scale [9] and generating several new items. The 16 items presented a choice between two alternatives 
according to three main criteria. First, each item presented one immediately available alternative and another academic alternative that could be obtained after a delay interval. Second, selecting the delayed academic alternative presumably increased the probability of long-term academic success. However, selecting the competing alternative produced immediate gratification but diminished the probability of long-term academic achievement. Third, the delayed academic alternative should be considered more valuable by the students than the immediately available alternative. A principle component analysis with varimax rotation of responses from 180 undergraduates on the 16-items scale retained one factor with 10 -items (i.e., academic delay of gratification). Six items were deleted because their item-total correlations were substantially low. Herndon [14] replicated the findings of Bembenutty \& Karabenick [13] using a sample of 319 middle school students and demonstrated that the ADOGS is a 10item unidimensional measure. In another study, Bembenutty \& Karabenick [10] reported that a principal component analysis with varimax rotation of responses from 194 undergraduates on the ADOGS retained two factors. The first factor consisted of six items (Items 1, 3, 4, 5, 6, 8) that presented choices between academic and non-social alternative activities (e.g., missing class because of nice weather). The second factor consisted of four items (Items $2,7,9,10)$ that presented choices between academic and social alternative activities (e.g., study little to spend more time with friends). Despite evidence of multidimensionality, Bembenutty and Karabenick treated the ADOGS as a unidimenional scale because the level of internal consistency did not support the use of subscales. Several studies have examined the validity of the factorial structure of the translated versions of the ADOGS [13]. For example, Villarroel [15] analyzed the responses from 120 undergraduates on the Spanish version of the ADOGS using confirmatory factor analysis (CFA). He reported that a hierarchical factor model that assumes the two correlated first-order factors (academic versus non-social activities and academic versus social activities) reflected a single; second-order factor of academic delay of gratification fitted the data adequately. King \& Du [32] found that a CFA of responses from 187 undergraduates on the Chinese version of the ADOGS showed that the unidimensional model fitted the data well only after correlating the error terms of Item 1 and Item 4 because both items share similar content (i.e., forgoing social events). A multigroup CFA demonstrated that the model was invariant across gender. Arabzadeh et al. [17] reported that a CFA of responses from 600 high school students on the Persian version of the ADOGS showed that a unidimensional model fitted the data well for the whole sample as well as for males and females separately. The authors did not report any findings on the invariance of the ADOGS across gender. More recently, Ganotice \& King [18] tested three alternative models of the Filipino version of ADOGS: (a) a global one-factor model that assumes all items reflect a single general academic delay of gratification factor, (2) a correlated two-factor model (i.e., academic and non-social alternative activities versus academic and social alternative activities), and (3) a three factor model wherein Factor 1 (academic vs. social alternatives) contained items 8 and 9; Factor 2 (academic vs. non-social alternatives) contained items 1, 3, 5, 6, and Factor 3 (reversed items) contained the two reversed items (items 2 and 7). Although the three models showed good fit to the data from 425 high school students, the authors supported the one factor model for parsimony purposes. The ADOGS showed partial invariance for the one-factor model across gender (Item 6 showed noninvariance) and complete invariance across year level.

\section{Gender differences on the ADOGS}

With respect to gender differences on the ADOGS, Bembenutty \& Karabenick [10] reported that female college students had higher levels of academic delay of gratification than their male counterparts. Likewise, Bembenutty [19] found that minority females had higher levels of academic delay of gratification than Caucasian males in a sample of college students. Bembenutty [20] reported that gender was a significant predictor of academic delay of gratification of college students with female students reporting higher levels of academic delay of gratification than their male counterparts. Villarroel [15], using a sample of Spanish undergraduates, found that females reported higher levels of academic delay of gratification than their male counterparts. In contrast, Bembenutty [21] found nonsignificant gender differences in academic delay of gratification in a sample of undergraduates. He also reported that the interaction effect between ethnicity and gender with regard to academic delay of gratification was not statistically significant.

\section{Academic delay of gratification and motivational determinants}

One important framework that proves to be helpful in explaining learners' preferences for diverse alternatives of action in an academic delay of gratification situation is the expectancyvalue theory [22-24]. In this theory, achievement outcomes, such as task performance and future aspirations, are primarily influenced by internalized perceptions of outcome expectancies and value of specific tasks or domains. The expectancy component corresponds to beliefs about one's own competence and selfefficacy. The value component refers to the reasons for engaging in a specific task and includes four principal components: attainment value, intrinsic value, utility value, and cost. Attainment value is defined as the personal importance of doing well on a task, whereas intrinsic value refers to the enjoyment an individual gets from performing an activity, or to the subjective interest an individual has in a subject or activity. Utility value is determined by how well a task or domain relates to current and future goals, such as career goals and academic aspirations. Finally, cost is conceptualized in terms of the negative aspects of engaging in a task, such as performance anxiety and fear of both failure and success, as well as the amount of effort needed to succeed and the lost opportunities that result from making a choice rather than another [22-25]. Mischel [26] has assessed motivational determinants of delay of gratification, such as the relevance, value, and expectancy for an immediate reward versus delayed reward options. His research has suggested that students' willingness to delay gratification depends upon the relative value placed on the competing alternatives. In addition, students' choice to delay gratification depends upon their expected likelihood of 
successful performance, given that they devote their time to this academic goal instead of a more immediate reward. Bembenutty and his colleagues [10-27] have demonstrated a relationship between academic delay of gratification and several motivational determinants. For example, Bembenutty [27] found that college students were more likely to engage in academic delay of gratification when they liked the delay alternative, considered the delay alternative more important than the non-delay alternatives, and had higher expectations that the delay alternative would provide better outcomes than the non-delay alternatives. Further, after controlling for gender, importance of the delay alternative versus immediate alternative was a significant predictor of academic delay of gratification.

\section{Academic delay of gratification and academic achievement}

There is compelling research evidence that individual differences in children's delay preferences are associated with subsequent higher academic achievement, intelligence, and the need for achievement [1-5]. Bembenutty \& Karabenick [10] reported that academic delay of gratification correlated positively with expected and obtained final course grades in a sample of college students. Bembenutty [19] found a positive relationship between academic delay of gratification and final course grade for Caucasian males and females college students. Bembenutty [20] demonstrated that the positive relationship between academic delay of gratification and academic achievement held even after controlling for the effect students' rating of the course, expected grade, and degree of interest, importance, and utility of the academic task.

\section{Academic delay of gratification and social desirability}

The society, in general, expects students to become responsible for their academic achievement and strive to remain goal oriented and committed to tasks for the sake of long-term valuable outcomes while facing distractions that are typical features of learning contexts [10]. In line with these expectations, individuals learn that certain kinds of responses are more desirable and that others are less desirable. The desirability of behaving within the confines set out by one's larger group is dependent on the strength of societal pressures with which one is confronted through his or her learning experiences. A possible effect of these societal pressures is that one will tend to omit the undesirable responses or check the more favorable responses on a self-reported measure [28]. Given this notion, it is not clear whether students' responses to the ADOGS items are free of social desirability and as such these responses reflect students' true levels of tendency to delay gratification, or whether these responses are affected by the students' tendency to respond in a socially desirable manner and as such these responses are an artifact methodology of social desirability bias.

\section{Aims and rationale of the present study}

There have been several calls in recent years for researchers within the area of educational and psychological assessment to be more sensitive to the cultural context $[29,30]$. The cultural variation in construing human behavior becomes more important for the study of academic delay of gratification because some cultures may be more conducive of academic delayed gratification than others. This may be true because cultures differ in the educational opportunities offered to students, the available attractive alternative activities that are typical features of learning contexts, and the values associated with delaying immediately available opportunities in favor of pursuing chosen important academic goals. For example, in societies with stronger collectivistic values, students have greater filial allegiances [31] that result in stronger pressures for high academic performance and academic delay of gratification [32]. Furthermore, we know little about the propensity of academic delay of gratification behavior among native Egyptian adolescents because the number of studies conducted using the ADOGS is limited. It is possible that the paucity of research on academic delay of gratification on Egyptian adolescents has, in part, been due to the lack of Arabic language measures with acceptable psychometric properties and also to the fact that many Egyptian adolescents do not have an adequate command of the English language for the use of English language questionnaires. Thus, there is a lack of an even more rapidly applicable and reliable measures of academic delay of gratification in the Arabic-speaking context; a tool not only valid but also useful for providing information about adolescents' academic delay of gratification. Doing so, it is also possible to facilitate and promote academic delay of gratification research with Arabic-speaking adolescents. In fact, the lack of Arabiclanguage measures with acceptable psychometric properties of academic delay of gratification could have several important implications. First, there are limited normative data to make clinical assessments of non-English speakers. Second, possible construct differences, interpretation problems, and response biases might have important effects on responses to questionnaires that are not written in the respondent's first language. Third, it is not possible to make reliable comparisons across cultures or to compare individuals within certain cultures if standardized instruments are unavailable or if different instruments are used in each culture. In line with this argument, the validity of the ADOGS [10-13] needs to be assessed in a non-Western context because it is possible that some instruments developed in the West might not work properly in non-Western settings due to cultural differences $[29,30]$. Specifically, the present study adopts a construct validation approach to the empirical assessment of the ADOGS. A construct validation approach utilizes both withinnetwork and between-network constructs validation. Withinnetwork construct validation explores the internal structure of a construct by employing statistical techniques such as exploratory factor analysis, CFA, and reliability analysis. On the other hand, the between-network validation approach explores the patterns of relationships between the construct and other theoretically related constructs by utilizing statistical techniques such as correlation, regression, or path analysis [22]. Thus, the aim of the present study is twofold:

a. To explore the within-network validity of the ADOGS by examining the consistency of item means and variances, item distributional properties, corrected item-total correlations, reliability coefficients with respective deletion of items, 
congeneric CFA loadings, the invariance of the ADOGS factorial structure across gender, and gender differences in conceptualizing academic delay of gratification.

b. To establish the between-network validity of the ADOGS by examine the relationship among academic delay of gratification, motivational determinants for the delay versus non-delay alternatives, academic achievement, and social desirability using correlation analysis.

The results from this study can contribute to the body of literature on academic delay of gratification by providing strong and necessary empirical evidence of construct, discriminant, and criterion-related validity, as well as validity of inferences regarding gender differences for Bembenutty \& Karabenick's [13] model of academic delay of gratification as articulated via the ADOGS. This study focuses on Egyptian adolescents because one of the main challenges encountered by adolescents is to resist temptations of non-academic alternatives and remain goal oriented and committed to academic tasks [10-33]. In addition, Egypt represents a collectivistic Middle Eastern country that has recently operated extensive development programs in education. There are some meaningful characteristics that might strengthen the cross-gender utility of the measurement model of academic delay of gratification as described in the ADOGS. Egypt is more open to the Western culture and is typically more liberal compared to many other Arab countries. The Egyptian culture provides adolescents with chances to test and develop their masculine and feminine social roles $[34,35]$.

\section{Methods}

\section{Participants}

Sample 1: A total of 450 Egyptian students (235 males and 215 females) from four public secondary schools in El-Minia governorate in Egypt participated in this study. The public education system in Egypt consists of basic education stage for those 4 to 14 years old (primary school for 6 years and preparatory school for 3 years), a secondary stage is for 3 years, for those ages 15 to 17 , and the tertiary level. All participant students were in their first year. All schools were from metropolitan areas and had single-gender populations (two female schools and two male schools). The mean sample ages were 16.29 (SD = .75) and 16.43 $(\mathrm{SD}=.54)$ for boys and girls, respectively. Only students with complete data were retained for this study. The percentage of students who had incomplete data was $2 \%$. Those students left several items blank on the ADOGS. The analysis of demographic data showed that all participant students were from the same ethnic background and that $98 \%$ of them were from working and lower class. Arabic was the native language of all participant students.

Sample 2: A total of 410 Egyptian students (215 males and 195 females) from four public secondary schools in El-Minia governorate in Egypt participated in this study. All participant students were in their second year. All schools were from metropolitan areas and had single-gender populations (two female schools and two male schools). The mean sample ages were $16.12(\mathrm{SD}=.48)$ and $16.25(\mathrm{SD}=.60)$ for boys and girls, respectively. Only students with complete data were retained for this study. The percentage of students who had incomplete data was $3 \%$. Those students left several items blank on the ADOGS. The analysis of demographic data showed that all participant students were from the same ethnic background and that $99 \%$ of them were from working and lower class. Arabic was the native language of all participant students. A $2 \times 2$ chi-square test with Yates's continuity correction revealed that the percentage of gender did not differ across both samples, $\chi 2(1, N=860)=.002$, $p$ $>0.852$, Phi ( ) effect size $=.002$.

\section{Measurements}

\section{Academic delay of gratification}

Bembenutty \& Karabenick [10] developed the 10-item ADOGS to assess college students' tendencies to delay gratification within specific academic situations. For each situation, the students first rated their preference for an option that offered immediate gratification such as "Miss several classes to accept an invitation for a very interesting trip" or a delay gratification option such as "Delay going on the trip until the course is over." Students responded to each item on a 4-point scale: Definitely choose A; Probably choose A; Probably choose B, or Definitely Choose B. The possible scores for the scale ranged from 10 to 40 . Higher scores were thus indicative of greater of academic delay of gratification. The authors, working without referencing to the English version of the ADOGS, independently translated ADOGS from English into Arabic using the back-translation method. Two other qualified translators, working without referencing to the English version of the ADOGS, independently compared the original English version of the ADOGS to the new English version that was translated back from Arabic, and rated the match between the two versions on a scale of 0 or 1 . A score of zero represented no match, whereas a score of 1 represented perfect match. The average percentage of match was $98 \%$ which could be considered highly acceptable [36]. Furthermore, interobserver agreement was calculated using SPSS 22.0 Crosstabs function, which produces a Kappa statistic for level of agreement. According to Cohen [37], Kappa values lie between -1.00 and 1.00, with zero indicating chance agreement, positive values indicating greater than chance agreement, and negative values indicating less than chance agreement. Landis \& Koch [38] categorized Kappa values from 0.41 to 0.60 as moderate and values above .60 as substantial levels of agreement. The interobserver agreement Kappa value for the ADOGS was .76 which indicated high levels of interobserver agreement.

\section{Motivational determinants}

Students were asked to report how strongly they agree or disagree with statements that describe motivation-related features of the delayed and immediately-available alternatives. These motivational-related features were as follows: Liking (e.g., "This is something that I would like to do"); Importance (e.g., "This is something that is important to me"); Expectancy (e.g., "This is something that would help me to achieve my academic goals"); Utility (e.g., "This is something that can be useful to me"), Negative Consequences (e.g., "This is something that can have negative 
consequences to me"), and Time/Effort (e.g., "This is something that can be costly in time or effort to me). Students responded to all items of the Motivational Determinants Scale (MDS) on a 4-point Likert-type scale ranging from 1 (Strongly Disagree) to 4 (Strongly agree). Difference scores between motivation for the delay and immediate preferences were obtained by subtracting responses to the immediate alternative from the delay alternative for the six items (e.g., liking of the delay alternative minus liking for the immediate alternative $=$ difference in liking). Higher scores were thus indicative of greater liking, importance, expectancy, utility, negative consequences, and time/effort for the delay versus non-delay alternatives.

\section{Academic achievement}

Students' academic achievement scores were obtained from their school records at the end of the academic year. These were the courses aggregated total scores, that is, the sum of on-course assignments and midterm and final examinations scores and were expressed as percentage.

\section{Social desirability}

Morad \& Al-Kaabi [39] translated the Marlowe-Crowne Social Desirability Scale-Short Form C [40] from English into Arabic using samples of high school students from Egypt, Algeria, and Oman. This is a 13-item single-factor measure. An example of the scale items is "It is sometimes hard for me to go on with my work if I am not encouraged." Students responded to each item on dichotomous yes-no scale. A yes response was assigned a score of 1 , indicating social desirability. A no response was assigned a score of 0 , indicating a socially undesirable response. The possible scores for the scale ranged from 0 to 13 . Higher scores were thus indicative of greater of social desirability.

\section{Procedure}

Approval was obtained to conduct the research investigation at the schools prior to data collection. Students were recruited to participate in the present study during their normal classes at their schools. All students gave assent by signing a consent form prior to their participation in the present study that they are willing to respond to the ADOGS, the MDS, and the MCSDS-Short Form C. The consent form also indicated that participant students agree that their end-of academic year achievement scores can be obtained from their school records. Students first responded to the ADOGS, then the MDS, and finally the MCSDS-Short Form C. The three measures were administered by trained experimenters according to standardized instructions. Students were informed that participation is voluntary and that confidentiality of their answers would prevail at all times. Only certain classes in each school participated in the present study depending on students' classroom schedules on the day and time of the administration of the measures. Apparently, the items of the Arabic version of the ADOGS were within the age-equivalent reading level of the Egyptian adolescents because they did not indicate any difficulty understanding their content. Students completed the three measures in 20 to 30 minutes. Students' academic achievement scores were obtained from students' school records at the end of the academic year.

\section{Overview of the statistical analysis}

\section{Factorial structure}

CFA was used to test five alternative factorial structures of the ADOGS as suggested in previous studies:

a. a global one-factor model that assumes all items reflect a single general academic delay of gratification factor [13-18].

b. A correlated two factor model of academic versus social alternatives activities (Factor 1 ) and academic versus nonsocial alternative activities (Factor 2) [10-18].

c. An uncorrelated two factor model of academic versus nonsocial alternatives activities and academic versus social alternative activities, implying an orthogonal solution [10].

d. A hierarchical model that assumes the two correlated firstorder factors (academic versus non-social activities and academic versus social activities) reflected a single, secondorder factor of academic delay of gratification [15].

e. A three factor model wherein Factor 1 (academic vs. social alternatives) contained items 8 and 9; Factor 2 (academic vs. non-social alternatives) contained items 1, 3, 5, 6, and Factor 3 (reversed items) contained the two reversed items (items 2 and 7) [18].

By comparing our hypothesized one-factor model with alternative models, we can be more confident of which model fits the data best. In the present study, Sample one was used to modify the model post hoc and the Sample 2 sample was used to cross-validate the model a priori to avoid being misled by the unique characteristics of a single sample [19]. Several absolute and relative goodness-of-fit indexes were used to evaluate each model's goodness-of-fit to the data. Absolute fit indices included Chi-square ( $\chi 2$ ), Standardized Root Mean-Square Residual (SRMR), and Root-Mean-Square Error of Approximation (RMSEA). Relative fit indices included Comparative Fit Index (CFI) and Nonnormed Fit Index (NNFI). When modeling normally distributed data, SRMR values of approximately .08 or below, RMSEA values of approximately .06 or below, CFI values of approximately .95 or above, and NNFI of approximately .95 or higher suggest adequate model-data fit [20-44]. Because the $\chi 2$ is sensitive to sample size, Hoelter [22] recommended reporting the $\chi 2 / \mathrm{df}$ ratio and suggested that ratios below 2.0 indicate a reasonable fit.

\section{Measurement invariance}

A decision was made a prior to follow a protocol for the steps of conducting the analysis of measurement invariance based on how the results of the analysis came out at each step in the process. If the same CFA model fits the data from the Egyptian samples well and fits statistically and significantly better than alternative models, additional invariance tests will be conducted to determine if the measure is functioning differentially across the two samples. If, however, a different structure is supported across the two samples, additional invariance testing will not be completed and the focus will turn instead to examine these differences in structure. The invariance testing process involves several steps in which increasingly restrictive levels of measurement invariance 
are explored. Four levels of measurement invariance will be tested in the following order:

a. Configural invariance.

b. Metric invariance.

c. Scalar invariance.

d. Latent mean analysis [48-60].

I. Configural invariance: Configural invariance tests whether the same basic factor structure holds for the two groups. This level of invariance tests only the overall structure: whether the same number of factors is relevant and whether the same items are salient to each factor across groups (i.e., same pattern of fixed and freed loadings). This model then served as a baseline model for comparisons with more restricted models.

II. Metric invariance: Metric invariance can be tested-if configural invariance is established- by constraining the matrix of factor loadings to be invariant across groups. Metric invariance is met if the strength of the relations between each item and its associated factor are equivalent across groups. If metric invariance is supported, one can conclude that the two groups of interest are interpreting the items in the same way. A lack of metric invariance may imply that some items are more important to the construct for one group than for the other. When full metric invariance is not established, the researcher can determine the source of the noninvariance by freeing, progressively, the loadings in the baseline model for items across the groups, until a final partial metric invariance model is obtained. This final partial metric invariance model will have only those items with equal loadings constrained equally across the groups. When a few items are found to be noninvariant, meaningful cross-group comparisons can still be performed because few items will not heavily influence such comparisons [46].

III. Scalar invariance: Scalar invariance tests the equality of intercept term by imposing an equality constraint on the intercepts of the items found to have invariant factor loadings (i.e., metric invariance). At this step, one is essentially testing to see whether the two groups use the response scale in a similar way.

For instance, a student from Sample 1 and a student form Sample 2 with the same latent level of academic delay of gratification should choose the same response option for an academic delay of gratification item if the academic delay of gratification item does in fact represent academic delay of gratification and functions similarly across the two samples. Scalar invariance is important because it must be satisfied before interpreting mean differences between the groups. If this initial model is not supported, the source of the non-invariance can be explored, using a similar strategy to that described for testing partial metric invariance. The final partial scalar invariance model can be revised appropriately to test for invariance in latent means. In general, for testing invariance for the structural model it is necessary for at least one item to show scalar invariance in addition to the loadings that are equal due to their being fixed at unity (e.g., 1) for identification purposes [47]. Because each of the increasingly constrained invariance models is nested within the previous models, the change in fit will be assessed by comparing fit indices from one step to the next. Typically, model comparisons are made by examining the change in $\chi 2$ relative to the change in the degrees of freedom of the two compared models [46]. However, additional indices have been recommended for comparing nested models. Therefore, in addition to the difference in $\chi 2(\Delta \chi 2)$, the change in model fit will be assessed by examining the change in the CFI index $(\Delta \mathrm{CFI})$. Decreases of .01 or less in the CFI index will be interpreted to suggest invariance at that step [48]. In the present study, the $\Delta$ CFI test was given more weight whenever there was disagreement between the conclusions of the $\Delta \times 2$ test and the $\Delta$ CFI test. According to Byrne [45], this is an arbitrary decision and rests solely with each individual researcher. However, Cheung \& Rensvold [48], in a Monte Carlo study of 20 different fit indices, reported that like the $\chi 2$ test, $\Delta \chi 2$ test is also susceptible to sample size and/or model complexity and has less value in making practical decisions about measurement invariance. They also, reported that $\Delta \mathrm{CFI} \leq 0.01$, was the best indication of support of measurement invariance. Wu et al. [49] has described Cheung \& Rensvold's [48] findings as " the most justifiable theoretically or empirically to date." (p.9)

IV. Latent mean analysis: If configural, metric, and scalar invariance were supported, one is able to estimate latent mean differences and effect sizes [49]. Byrne et al. [48] proposed that latent means can still be compared under partial intercept invariance assuming that the latent means will not be affected by noninvariant intercepts to a great extent. A structured means modeling approach was chosen over multivariate analysis of variance (MANOVA) because in MANOVA, group differences are assessed on the linear composites of measured variables while including the measurement error of variables in the composite. On the other hand, structural equation modeling approaches allow for the use of theoretically error-free constructs in tests of group differences. Therefore, they are more powerful than MANOVA approaches as they parse out the measurement error [51].

Correlational analysis: We used Pearson's correlation to examine the relationships among academic delay of gratification, motivational determinants, academic achievement, and social desirability.

\section{Results}

\section{Data screening}

Means, standard deviations, skewness, kurtosis, and item-total correlations of the ADOGS items for males and females within Sample 1 and Sample 2 are summarized in Table 1. Based on the analyses conducted using the DeCarlo [52] macro, there were no significant multivariate outliers. The variance inflation factor and the tolerance values were 2.33 and .96 respectively, suggesting that there was no significant multicollinearity in the data. Normality was assessed both univariately and multivariately. 
Absolute values of skewness and kurtosis larger than 2 and 7, respectively, might imply a lack of univariate normality [53]. Table 1 shows that the values of univariate skew and kurtosis of all variables were within acceptable ranges, suggesting that the data have normal univariate distributions. The multivariate kurtosis equaled 2.23. Whereas there is no standard cutoff for this index, Kline [54] recommended that multivariate normality can be assumed if this value is less than 3. Given that the data appear normally distributed at univariate and multivariate levels, the full information maximum likelihood estimation was used to analyze the variance-covariance matrices and estimate model parameters and obtain fit indexes in all CFA analyses [45]. The AMOS 7.0 program [55] was used to run all analyses (Table 1).

\section{Factorial structure}

CFA results, summarized in Table 2, showed that the unidimensional model had a close fit to the data of both Egyptian samples. The item loadings for Sample 1 ranged from .65 to .78 and the error terms ranged from .33 to .44. The item loadings for Sample 2 ranged from .61 to .74 and the error terms ranged from .35 to .43 . However, the correlated two-factor model, the uncorrelated (orthogonal) two factor model, the hierarchical model, and three factor model had goodness-of-fit statistics that indicated inadequate fit to the data of both samples. These findings support the unidimensionality of the ADOGS. The unidimensional model of the ADOGS was used in further analyses (Table 2).

Table 1 :Descriptive statistics of the 10-items of the ADOGS by gender and sample.

\begin{tabular}{|c|c|c|c|c|c|c|}
\hline Items & Gender & Mean & SD & Skewness & Kurtosis & Item-Total Correlations \\
\hline \multicolumn{7}{|c|}{ Sample $1(\alpha=.85)$} \\
\hline \multirow{2}{*}{ Item 1} & Males & 2.62 & 0.36 & -0.37 & 1.26 & 0.7 \\
\hline & Females & 2.51 & 0.27 & 0.3 & 1.12 & 0.74 \\
\hline \multirow{2}{*}{ Item 2} & Males & 3.15 & 0.16 & 0.47 & 1.55 & 0.71 \\
\hline & Females & 2.93 & 0.22 & 0.55 & 1.68 & 0.68 \\
\hline \multirow{2}{*}{ Item 3} & Males & 2.75 & 0.59 & 0.34 & -0.77 & 0.72 \\
\hline & Females & 2.63 & 0.67 & 0.45 & 0.54 & 0.75 \\
\hline \multirow{2}{*}{ Item 4} & Males & 2.81 & 0.41 & 0.5 & .1 .10 & 0.67 \\
\hline & Females & 2.45 & 0.34 & -0.44 & -0.97 & 0.65 \\
\hline \multirow{2}{*}{ Item 5} & Males & 2.5 & 0.78 & 1.23 & 0.76 & 0.75 \\
\hline & Females & 2.62 & 0.73 & 1.3 & -70 & 0.73 \\
\hline \multirow{2}{*}{ Item 6} & Males & 3.3 & 0.39 & -0.56 & 1.41 & 0.68 \\
\hline & Females & 3.45 & 0.32 & 0.66 & 1.22 & 0.66 \\
\hline \multirow{2}{*}{ Item 7} & Males & 2.22 & 0.47 & 1.37 & 1.22 & 0.64 \\
\hline & Females & 2.36 & 0.42 & 1.44 & -1.04 & 0.63 \\
\hline \multirow{2}{*}{ Item 8} & Males & 2.68 & 0.55 & 0.29 & 0.75 & 0.66 \\
\hline & Females & 2.89 & 0.46 & 0.22 & 0.8 & 0.65 \\
\hline \multirow{2}{*}{ Item 9} & Males & 3.11 & 0.37 & -0.41 & 0.7 & 0.73 \\
\hline & Females & 3.35 & 0.24 & -0.35 & 0.73 & 0.7 \\
\hline \multirow{2}{*}{ Item 10} & Males & 2.9 & 0.31 & 0.55 & 1.15 & 0.71 \\
\hline & Females & 2.67 & 0.4 & 0.67 & 1.31 & 0.74 \\
\hline \multicolumn{7}{|c|}{ Sample $2(\alpha=.87)$} \\
\hline \multirow{2}{*}{ Item 1} & Males & 2.39 & 0.39 & 0.45 & 0.69 & 0.74 \\
\hline & Females & 2.19 & 0.31 & -0.39 & 0.75 & 0.73 \\
\hline \multirow{2}{*}{ Item 2} & Males & 2.64 & 0.23 & 1.3 & 1.23 & 0.73 \\
\hline & Females & 2.46 & 0.3 & 1.25 & 1.3 & 0.69 \\
\hline \multirow{2}{*}{ Item 3} & Males & 2.33 & 0.64 & 0.6 & -0.85 & 0.76 \\
\hline & Females & 2.52 & 0.57 & 0.53 & -0.93 & 0.77 \\
\hline \multirow{2}{*}{ Item 4} & Males & 3.36 & 0.66 & 1.29 & 1.4 & 0.69 \\
\hline & Females & 3.2 & 0.72 & 1.23 & 1.53 & 0.66 \\
\hline
\end{tabular}




\begin{tabular}{|c|c|c|c|c|c|c|}
\hline \multirow{3}{*}{ Item 5 } & Males & 2.59 & 0.3 & 0.52 & 0.55 & 0.63 \\
\cline { 2 - 7 } & Females & 2.3 & 0.49 & 0.45 & 0.46 & 0.61 \\
\hline \multirow{3}{*}{ Item 6 } & Males & 2.99 & 0.6 & 0.39 & 1.1 & 0.66 \\
\cline { 2 - 7 } & Females & 3.18 & 0.48 & 0.33 & 1.22 & 0.69 \\
\hline \multirow{2}{*}{ Item 7 } & Males & 3.14 & 0.65 & 1.3 & 1.53 & 0.65 \\
\cline { 2 - 7 } & Females & 3.45 & 0.6 & 1.38 & 1.37 & 0.64 \\
\hline \multirow{2}{*}{ Item 8 } & Males & 3.11 & 0.76 & 0.24 & 0.64 & 0.75 \\
\cline { 2 - 7 } & Females & 3.38 & 0.73 & 0.29 & 0.57 & 0.73 \\
\cline { 2 - 7 } & Males & 2.75 & 0.66 & 1.19 & 1.52 & 0.72 \\
\hline \multirow{2}{*}{ Item 9 } & Females & 2.58 & 0.52 & 1.26 & 1.4 & 0.75 \\
\hline & Males & 2.63 & 0.46 & 0.44 & 0.98 & 0.67 \\
\hline
\end{tabular}

Note. $N=450$ (235 males and 215 females) and 410 (215 males and 195 females) for Sample 1 and Sample 2 respectively.

Table 2: Goodness-of-fit statistics for four alternative measurement models of the ADOGS across Sample 1 and Sample 2.

\begin{tabular}{|c|c|c|c|c|c|c|c|c|}
\hline Model & Sample & $\chi 2$ (df) & df & $\chi^{2} / \mathrm{df}$ & SRMR & RMSEA & CFI & NNFI \\
\hline \multirow{2}{*}{ One-factor model } & Sample 1 & 63.69 & 35 & 1.81 & 0.04 & .04 (CI: .02-.07) & 0.97 & 0.98 \\
\hline & Sample 2 & 57.24 & 35 & 1.63 & 0.04 & .03 (CI: .01-.05) & 0.97 & 0.98 \\
\hline \multirow{2}{*}{ Correlated two-factor model } & Sample 1 & 114.88 & 34 & 3.37 & 0.12 & .15 (CI: .09-.19) & 0.88 & 0.87 \\
\hline & Sample 2 & 122.82 & 34 & 3.61 & 0.14 & .14 (CI: .08-.18) & 0.89 & 0.89 \\
\hline \multirow{2}{*}{ Uncorrelated two-factor model } & Sample 1 & 145.36 & 35 & 4.15 & 0.16 & .22 (CI: .15-.28) & 0.77 & 0.81 \\
\hline & Sample 2 & 131.73 & 35 & 3.76 & 0.17 & .19 (CI: .12-.25) & 0.75 & 0.82 \\
\hline \multirow{2}{*}{ Hierarchal model } & Sample 1 & 145.36 & 34 & 4.27 & 0.14 & .14 (CI: .10-.19) & 0.75 & 0.8 \\
\hline & Sample 2 & 131.73 & 34 & 3.87 & 0.16 & .13 (CI: .08-.19) & 0.75 & 0.79 \\
\hline \multirow{2}{*}{ Three factor model } & Sample 1 & 165.22 & 32 & 5.16 & 0.18 & .16 (CI: .10-.21) & 0.73 & 0.75 \\
\hline & Sample 2 & 152.13 & 32 & 4.75 & 0.16 & .17 (CI: .12-.24) & 0.72 & 0.73 \\
\hline
\end{tabular}

Note. $N=450$ and 410 for Sample 1 and Sample 2 respectively. CI represents 90\% confidence interval. SRMR: Standardized Root Mean Square Residual; RMSEA: Root Mean Square Error of Approximation; CFI: Comparative Fit Index; NNFI: Nonnormed Fit Index

\section{Gender invariance}

Tests for measurement invariance were conducted across gender within each sample (i.e., Sample 1 males were compared with Sample1 females and Sample 2 males were compared with Sample 2 females) to examine the generalizability of the unidimensional model of the ADOGS across gender in Sample 1 to Sample 2.

\section{Sample 1}

a. Configural invariance: Table 3 shows that the ADOGS model fit the data reasonably well for males and females across both Sample 1 and Sample 2.

b. Metric invariance: We tested a model in which the item loadings on their designated factor were constrained to be equal for males and females. Table 3 shows that the difference between the unconstrained model and the constrained model was not significant. Also, the change in the CFI was less than the cutoff score of .01. c. Scalar invariance: We tested scalar invariance by constraining the intercepts of all items of the ADOGS. Table 3 shows that the difference between the unconstrained model and the constrained model was not significant. The change in the CFI was less than the cutoff score of .01.

d. Latent mean analysis: In latent mean analysis, the mean of a latent variable cannot be estimated directly. However, it is possible to estimate the difference between the means of a latent variable across groups by fixing one of these means to zero in one of the groups (i.e., the reference group). Accordingly, the estimated value of the latent mean in the other group indicates the latent mean difference between the two groups. The significance test for the latent mean estimate is the test for significance of the latent mean difference between the two groups. The effect sizes of latent mean difference between the two groups were calculated from: Effect size $=(\mathrm{M} 2-\mathrm{M} 1) / \mathrm{SD}, \mathrm{SD}=$ where $\mathrm{M}$ is latent variable estimated mean, $\mathrm{SD}$ is the standard deviation, $\mathrm{N}$ is 
the sample size, $\mathrm{V}$ is a latent variable estimated variance, and the subscripts denote groups [56]. Values of 0.2, 0.5, and 0.8 were considered small, medium, and large effects, respectively [57]. In this latent mean analysis, males were set as the reference group. The analysis showed that females had higher levels of academic delay of gratifications than males (Females $=.72, \mathrm{t}=3.40$, effect size $=.61)$.

\section{Sample 2}

a. Configural invariance: Table 3 shows that the ADOGS model fit the data reasonably well for males and females.

b. Metric invariance: Table 3 shows that the difference between the unconstrained model and the constrained model was marginally significant. The differences in CFI between the two models were less than the cutoff score of .01. As indicated earlier, the $\Delta \mathrm{CFI}$ test was given more weight whenever there was disagreement between the conclusions of the $\Delta \chi 2$ test and the $\Delta$ CFI test $[48,49]$.

c. Scalar invariance: Scalar invariance was tested by constraining the intercepts of all items of the ADOGS. Table 3 shows that the difference between the unconstrained model and the constrained model was not significant. Also, the change in the CFI was less than the cutoff score of .01. d. Latent mean analysis: Male group was set as a reference group. The analysis showed that females had higher levels of academic delay of gratifications than males (Females $=.66, \mathrm{t}$ $=3.22$, effect size $=.56$ ) (Table 3 ).

\section{Correlation analysis}

Table 4 shows Pearson's correlation coefficients among academic delay of gratification, liking, importance, expectancy, utility, negative consequences, time/effort, academic achievement, and social desirability for Sample 1 and Sample 2. Academic delay of gratification correlated significantly $(p<.01)$ with all motivational determinants in both samples. The correlation coefficients ranged from - .40 to .51 for Sample 1 and from .41 to .48 for Sample 2. Specifically, academic delay of gratification correlated positively with liking, importance, expectancy, and utility and negatively with negative consequences and time/ effort for the delay versus non-delay alternatives in both samples. Academic delay of gratification correlated positively and significantly $(\mathrm{p}<.01)$ with academic achievement in Sample $1(\mathrm{r}$ $=.49)$ and Sample $2(r=.46)$ samples. However, academic delay of gratification did not correlate significantly $(p>.05)$ with selfreported social desirability in Sample $1(\mathrm{r}=.08)$ or Sample $2(\mathrm{r}=$ .06) (Table 4).

Table 3: Results of measurement invariance tests for males and females within Sample 1 and Sample 2.

\begin{tabular}{|c|c|c|c|c|c|c|c|c|c|c|}
\hline Sample 1 & $\chi^{2}$ & df & $\chi^{2} / \mathbf{d f}$ & $\Delta \chi^{2}$ & $\Delta \mathbf{d f}$ & CFI & $\Delta \mathrm{CFI}$ & RMSEA & SRMR & NNFI \\
\hline $\begin{array}{l}\text { Configural: Factor structure } \\
\text { constrained to be equal }\end{array}$ & 108.22 & 70 & 1.55 & - & - & 0.979 & - & .04 (CI: .02 - .07) & 0.05 & 0.98 \\
\hline $\begin{array}{l}\text { Metric: Factor loadings constrained to } \\
\text { be equal }\end{array}$ & 122.29 & 79 & 1.62 & $13.07 \dagger$ & 9 & 0.971 & 0.008 & .04 (CI: .01 - .08) & 0.07 & 0.96 \\
\hline $\begin{array}{c}\text { Scalar: Intercepts constrained to be } \\
\text { equal }\end{array}$ & 134.47 & 88 & 1.54 & $26.25 \dagger$ & 18 & 0.973 & 0.006 & .03 (CI: .01 - .08) & 0.06 & 0.97 \\
\hline \multicolumn{11}{|l|}{ Sample 2} \\
\hline $\begin{array}{l}\text { Configural: Factor structure } \\
\text { constrained to be equal }\end{array}$ & 110.41 & 70 & 1.57 & - & - & 0.974 & - & .03 (CI: .01 - .07) & 0.05 & 0.97 \\
\hline $\begin{array}{l}\text { Metric: Factor loadings constrained to } \\
\text { be equal }\end{array}$ & 127.21 & 79 & 1.61 & $16.80^{*}$ & 9 & 0.967 & 0.007 & .04 (CI: .01 - .08) & 0.06 & 0.98 \\
\hline $\begin{array}{c}\text { Scalar: Intercepts constrained to be } \\
\text { equal }\end{array}$ & 137.51 & 88 & 1.56 & $27.10 \dagger$ & 18 & 0.968 & 0.006 & .04 (CI: .01 - .08) & 0.06 & 0.98 \\
\hline
\end{tabular}

Note. Male $=240$ and 215 and females $=220$ and 195 for Sample 1 and Sample 2 respectively. CI represents 90\% confidence interval. SRMR: Standardized Root Mean Square Residual; RMSEA: Root Mean Square Error of Approximation; CFI: Comparative Fit Index; NNFI: Nonnormed Fit Index ${ }^{*} p<.05 .{ }^{\dagger} p>.05$

Table 4: Correlations among academic delay of gratification, liking, importance, expectancy, utility, negative consequences, time/effort, academic achievement, and social desirability for Sample 1 (below diagonal) and Sample 2 (above diagonal).

\begin{tabular}{|c|c|c|c|c|c|c|c|c|c|}
\hline Variables & $\begin{array}{l}\text { Academic Delay } \\
\text { of Gratification }\end{array}$ & Liking & Importance & Expectancy & Utility & $\begin{array}{c}\text { Negative } \\
\text { Consequences }\end{array}$ & $\begin{array}{l}\text { Time/ } \\
\text { Effort }\end{array}$ & $\begin{array}{c}\text { Academic } \\
\text { Achievement }\end{array}$ & $\begin{array}{c}\text { Social } \\
\text { Desirability }\end{array}$ \\
\hline $\begin{array}{l}\text { Academic delay of } \\
\text { gratification }\end{array}$ & - & $.43^{* *}$ & $.45^{* *}$ & $.48^{* *}$ & $.41^{* *}$ & $-.42^{* *}$ & $-.44^{* *}$ & $.46^{* *}$ & 0.06 \\
\hline Liking & $.47^{* *}$ & - & $.35^{* *}$ & $.37^{* *}$ & $.41^{* *}$ & $-.34^{* *}$ & $-.29 *$ & $.40^{* *}$ & 0.04 \\
\hline Importance & $.44^{* *}$ & $.36^{* *}$ & - & $.34^{* *}$ & $.37^{* *}$ & $-.35^{* *}$ & $-.30 * *$ & $.41^{* *}$ & 0.1 \\
\hline
\end{tabular}




\begin{tabular}{|c|c|c|c|c|c|c|c|c|c|}
\hline Expectancy & $.51^{* *}$ & $.34^{* *}$ & $.36^{* *}$ & - & $.27 *$ & $-.35^{* *}$ & $-.30^{* *}$ & $.37^{* *}$ & 0.07 \\
\hline Utility & $.45^{* *}$ & $.38^{* *}$ & $.39 * *$ & $.31^{* *}$ & - & $-.29 *$ & $-.33^{* *}$ & $.35^{* *}$ & 0.09 \\
\hline $\begin{array}{c}\text { Negative } \\
\text { consequences }\end{array}$ & $-.40^{* *}$ & $-.30^{* *}$ & $-.41^{* *}$ & $-.32^{* *}$ & $-.29^{*}$ & - & $.27^{*}$ & $-.33^{* *}$ & 0.03 \\
\hline Time/effort & $-.42^{* *}$ & $-.32 * *$ & $-.33^{* *}$ & $-.35^{* *}$ & $-.32 * *$ & $.31^{* *}$ & - & $-.34 * *$ & 0.12 \\
\hline $\begin{array}{c}\text { Academic } \\
\text { achievement }\end{array}$ & $.49^{* *}$ & $.36^{* *}$ & $.37^{* *}$ & $.40^{* *}$ & $.38^{* *}$ & $-.35^{* *}$ & $-.37^{* *}$ & - & 0.13 \\
\hline Social desirability & 0.08 & 0.1 & 0.04 & 0.11 & 0.12 & 0.13 & 0.09 & 0.07 & - \\
\hline
\end{tabular}

Note. $N=450$ and 410 for Sample 1 and Sample 2 respectively. ${ }^{* *} p<.01,{ }^{*} p<.05$

\section{Discussion}

One important finding of this study is that the ADOGS singlefactor structure fit the data from two Egyptian samples well, and that none of the alternative models demonstrated adequate model-data fit in either sample. This finding indicates that the Arabic version of the ADOGS preserves the conceptual content of Bembenutty \& Karabenick's [10] original academic delay of gratification model. This pattern of results is consistent with Bembenutty \& Karabenick's [10] original findings concerning the factorial structure of the ADOGS. It also replicates the findings from research conducted internationally in China [16], United States [14], Iran [17], and Philippines [19]. The CFA also showed that the hierarchical model failed to achieve adequate fit to these data although Villarroel [15] argued that the hierarchical model could have acceptable fit. Likewise, the correlated two factor model [10-19], the uncorrelated two factor model [10], and the three-factor model [19] failed to achieve adequate fit to the data in either sample. Collectively, these findings support the unidimensionality of the ADOGS and indicate that the singlefactor model is an acceptable measurement model for the ADOGS as evidenced by appropriate goodness-of-fit statistics across diverse cultures. In relation to the evidences of convergent validity, Pearson's correlations were performed with the ADOGS and other measures of motivational determinants and academic achievement in both Egyptian samples. Self-reported academic delay of gratification correlated significantly and positively with liking, importance, expectancy, and utility, and negatively with negative consequences and time/effort. This finding firmly embeds academic delay of gratification within the framework of the expectancy-value theory [26,27] and the motivational view of delay of gratification [30]. Within hindsight, this appears to be a readily understood relationship: students' willingness to delay gratification in order to pursue long-term academic goals is associated with their motivation-related judgments of delay vs. non-delay alternatives as articulated by incentive value, benefits, or rewards associated with the academic alternatives and the tempting alternatives. Thus, an expectancy-value mechanism seems to underlie the subjective calculation and ultimately the decision of whether the value and feasibility of attaining a delayed reward relative to the value of the immediately available one is high enough to warrant choice to wait or work to attain it. This finding is consistent with the findings of Bembenutty and his colleagues [10-25]. For example, Bembenutty [23] reported that value based incentives were positively associated with how important, useful, and interesting college students perceived the delay alternative to be, but were inversely related to students' consideration of negative consequences associated with possible selection of the immediate alternatives versus delay alternatives. Furthermore, self-reported academic delay of gratification correlated significantly and positively with academic achievement. This relationship can be explained within the framework of the self-regulated learning theory because academic delay of gratification has commonly been conceptualized as involving successful self-regulated learning [10-58]. According to Bembenutty [23,24], when students engage in academic delay of gratification by deferring attractive activities in order to achieve long-term goals, they orchestrate their academics and self-impose constraints onto their own actions to facilitate and increase their academic achievement. Consistent with this notion, Mischel [30] conceptualized the ability to delay gratification as part of the selfregulatory system necessary to guide behavior without external controlling stimuli. He suggested that the ability to delay gratification is a process of self-regulatory system of willpower that orchestrates maintenance of motivation, engagement in goals, and ultimately an increase of academic achievement. In line with this finding, Bembenutty and his colleagues reported a positive relationship between academic delay of gratification and academic achievement [10-24]. Our data further demonstrated that self-reported academic delay of gratification did not correlate significantly with social desirability in both samples. This finding supports the discriminant validity of the ADOGS and highlights the notion that students' responses to the ADOGS are not biased by the need to respond in a culturally appropriate and acceptable manner. The multigroup CFA revealed that the ADOGS singlefactor structure was applicable for males and females within Sample 1 In line with these findings, King \& Du [16] reported that the ADOGS unidimensional model was invariant across gender. However, Ganotice \& King [19] found that a global one-factor model of the ADOGS showed partial invariance across gender. The configural invariance indicates that individuals within the compared groups have the same basic conceptualization of academic delay of gratification. More specifically, the satisfaction of configural invariance implies that individuals within the compared groups viewed the same items as salient to the measurement of academic delay of gratification. The achievement 
of metric invariance suggests that individuals within the compared groups might be using similar conceptual frames of reference when responding to all items of the ADOGS. That is, items have equal salience for individuals within the compared groups. The attainment of scalar invariance highlights that individuals within compared groups use the response scale in a similar way [16-58]. Overall, these findings increase our confidence in the external validity of ADOGS. Furthermore, these findings provide additional evidence for the ADOGS construct validity for the purpose of examining academic delay of gratification among Egyptian adolescents and for making cross-gender group comparisons. As suggested in previous literature, if measurement invariance has not been supported, valid cross-gender comparisons cannot be made [15-60]. Our data also demonstrated that when males and females were compared within both samples, females were higher in academic delay of gratification than males in both samples. This pattern of results appears to apply also to Western [9-23] as well as Asian college samples [16-19]. This finding is noteworthy since it suggests that males and females can show differences in the way they manage academic situations by remain goal oriented and committed to tasks while facing distractions that are typical features of learning contexts. However, this finding should not be interpreted as suggesting that males and females are inherently different in ways that lead them to engage in academic endeavors in a particular way. The socialization process and classroom contexts, including academic tasks, reward structures, instructional methods, and instructors' behaviors, may be associated with the patterns of the academic behavior reported by the students in the present study. However, this finding is in contrast with the finding of Bembenutty [25] who reported nonsignificant gender differences in academic delay of gratification in a sample of college students. He also found that the interaction effect between ethnicity and gender with regard to academic delay of gratification was not statistically significant. Our research conclusions are constrained by three important limitations. Firstly, we used self-report measures of motivational determinants and social desirability to collect criterion and discriminant information on the ADOGS. Although self-ratings of these constructs remain the standard used by most studies, future studies should assess behaviors associated with these constructs either as observed by others or by direct observations by researchers to increase confidence that the ADOGS actually measures academic delay of gratification. Until such studies are conducted, these constructs will remain largely defined as a cognitive self-construal processes rather than observable traits. Secondly, our data is of cross-sectional and as consequently definitive conclusions about the relationship among academic delay of gratification, motivational determinants, academic achievement, and social desirability cannot be drawn. A different method for understanding the developmental precursors and consequences of academic delay of gratification would be to examine them over time rather than at a single time point. Thirdly, the analyses were based on data collected from high school students only in Egypt, and therefore our results might not generalize to younger or older populations. In summary, the development of the Arabic version of the ADOGS is one of the strengths of this study. The findings demonstrated that the ADOGS had acceptable psychometric properties as a means of measuring academic delay of gratification. This measure functioned well and equivalently across gender. Although future studies are needed to replicate these results in additional settings, our findings suggest that researchers and practitioners can be more confident in their interpretation of ADOGS scores when used with culturally diverse samples.

\section{References}

1. Mischel W (1961) Preference for delayed reinforcement and social responsibility. Journal of Abnormal and Social Psychology 62(1): 1-7.

2. Mischel W, Ebbesen EB, Zeiss AR (1972) Cognitive and attentional mechanisms in delay of gratification. Journal of Personality and Social Psychology 21(2): 204-218.

3. Mischel W (1981) Metacognition and the rules of delay. In: Flavell JH \& Ross L (Eds.), Social cognitive development: Frontiers and possible futures. Cambridge University Press, England, UK, pp. 240- 271.

4. Mischel W, Metzner R (1962) Preference for delayed reward as a function of age, intelligence, and length of delay interval. J Abnorm Soc Psychol 64: 425-431.

5. Mischel W, Shoda Y, Peake PK (1988) The nature of adolescent competencies predicted by preschool delay of gratification. J Pers Soc Psychol 54(4): 687-696.

6. Funder DC, Block JH, Block J (1983) Delay of gratification: Some longitudinal personality correlates. J Pers Soc Psychol 44(6): 11981213.

7. Funder DC, Block JH (1989) The role of ego-control, ego resiliency, and IQ in delay of gratification in adolescence. J Pers Soc Psychol 57(6): 1041-1050.

8. Ray JJ, Najman JM (1986) The generalizability of deferment of gratification. Journal of Social Psychology 126: 117-119.

9. Ward WE, Perry TB, Woltz J, Doolin E (1989) Delay of gratification among Black college student leaders. Journal of Black Psychology 15: 111-118.

10. Bembenutty H, Karabenick SA (1998) Academic delay of gratification. Learning and Individual Differences 10: 329-346.

11. Pintrich PR, De Groot EV (1990) Motivational and self-regulated learning components of classroom academic performance. Journal of Educational Psychology 82(1): 33-40.

12. Pintrich PR, Schrauben B (1994) Students motivational beliefs and their cognitive engagement in classroom academic tasks. In: Schunk D \& Meece J (Eds.), Student perceptions in the classroom: Causes and consequences. Hillsdale, USA, pp. 149-184.

13. Bembenutty H, Karabenick SA (1996) Academic Delay of Gratification Scale: A new of measurement of delay of gratification. Paper presented at the Eastern Psychological Association, USA, pp. 1-11.

14. Herndon JS (2011) The effects of delay of gratification on the academic achievement, substance abuse, and violent behavior of middle school students in alternative learning settings. Unpublished doctoral dissertation, University of Central Florida, USA.

15. Villarroel JR (2008) An examination of the psychometric properties of the Spanish version of the Academic Delay of Gratification Scale. Advances in Applied Developmental Psychology 2: 156-167. 
16. King RB, Du H (2011) All good things come to those who wait: Validating the Chinese version of the Academic Delay of Gratification Scale (ADOGS). The International Journal of Educational and Psychological Assessment 7(1): 64-80.

17. Arabzadeh M, Kadivar P, Delavar A, Kavousian J (2012) Reliability, validity, and factor analysis of the Persian Academic Delay of Gratification Scale. Interdisciplinary Journal of Contemporary Research in Business 4(2): 571-579.

18. Ganotice FA, King RB (2014) Blessed are Those Who Wait: Validating the Filipino Version of the Academic Delay of Gratification Scale (ADOGS). The Asia-Pacific Education Researcher 23(1): 19-27.

19. MacCallum RC, Roznowski M, Necowitz, LB (1992) Model modification in covariance structure analysis: The problem of capitalization on chance. Psychol Bull 111(3): 490-504.

20. $\mathrm{Hu}$ LT, Bentler PM (1998) Fit indices in covariance structure modeling: Sensitivity to underparameterized model misspecification. Psychological Methods 3(4): 424-453.

21. Vandenberg RJ, Lance CE (2000) A review and synthesis of the measurements invariance literature: Suggestions, practices, and recommendations for organizational research. Organizational Research Methods 3: 4-69.

22. Hoelter JW (1983) The analysis of covariance structures: Goodnessof-fit indices. Sociological Methods and Research 11(3): 325-344.

23. Bembenutty $H$ (2007) Self-regulation of learning and academic delay of gratification: Gender and ethnic differences among college students. Journal of Advanced Academics 18(4): 586-616.

24. Bembenutty H (2009) Academic delay of gratification, self-regulation of learning, gender differences, and expectancy value. Personality and Individual Differences 46: 347-352.

25. Bembenutty H (1999) Sustaining motivation and academic goals: The role of academic delay of gratification. Learning and Individual Differences 11: 233-257.

26. Eccles JS (2005) Subjective task value and the Eccles et al. model of achievement related choices. In AJ Elliot \& CS Dweck (Eds.), Handbook of competence and motivation. Guilford Press, New York, USA, pp. 105-121.

27. Eccles JS (2007) Motivational perspective on school achievement: Taking responsibility for learning and teaching. In: R J Sternberg \& RF Subotnik (Eds.), Optimizing student success in schools with the new three Rs, Information Age, North Carolina USA, pp. 199-202.

28. Wigfield A, Eccles JS (2000) Expectancy-value theory of achievement motivation. Contemporary Educational Psychology, 25(1): 68-81.

29. Eccles JS, Wigfield A (2002) Motivational beliefs, values, and goals. Annu Rev Psychol 53: 109-132.

30. Mischel W (1996) From good intentions to willpower. In: Gollwitzer PM \& Bargh JA (Eds.), The psychology of action: Linking cognitions and motivation to behavior. Guilford Press, USA, pp. 197-218.

31. Bembenutty $H$ (2008) Academic delay of gratification and expectancy-value. Personality and Individual Differences 44: 193202.

32. Van de Mortel TF (2008) Faking it: Social desirability response bias in self-report research. Australian Journal of Advanced Nursing, 25(4): 40-48.
33. Basterra MR, Trumbull E, Solano Flores (2010) Cultural validity in assessment: Addressing linguistic and cultural diversity. Routledge, New York, USA.

34. Abd-El-Fattah SM (2013) A cross-cultural examination of the Aggression Questionnaire-Short Form among Egyptian and Omani adolescents. J Pers Assess 95(5): 539-548.

35. Abd-El-Fattah SM, Fakhroo HA (2012) The relationship among paternal psychological control and adolescents' perfectionism and self-esteem: A partial least squares path analysis. Psych 3(5): 428439.

36. Ratner C, Hui L (2003) Theoretical and methodological problems in cross-cultural psychology. Journal for the Theory of Social Behavior 33(1): 67-94.

37. Abd-El-Fattah SM, Patrick R (2011) The relationship among achievement motivation orientations, achievement goals, and academic achievement and interest: A multiple mediation analysis. Australian Journal of Educational and Developmental Psychology 11: 91-110.

38. Hofstede G (2001) Culture's consequences: Comparing values, behaviors, institutions and organizations across nations, Sage, USA, pp. 1-616.

39. Nydell MK (2005) Understanding Arabs: A guide for modern times. ( $4^{\text {th }}$ edn), Nicholas Brealey, England, UK, pp. 1-228.

40. Maneesriwongul W, Dixon JK (2004) Instrument translation process: A methods review. J Adv Nurs 48(2): 175-186.

41. Cohen J (1960) A coefficient of agreement for nominal scales. Educational and Psychological Measurement 20(1): 37-46.

42. Landis JR, Koch GG (1977) The measurement of observer agreement for categorical data, Biometrics 33(1): 159-174.

43. Morad GA, Al-Kaabi AK (2005) Psychometric properties short forms of the Marlowe-Crowne Social Desirability Scale. Algerian Journal of Psychology 13: 52-69.

44. Reynolds WM (1982) Development of reliable and valid short forms of the Marlowe-Crowne Social Desirability Scale. Journal of Clinical Psychology 38(1): 119-125.

45. Byrne BM (2010) Structural equation modeling with AMOS: Basic concepts, applications, and programming. ( $2^{\text {nd }}$ edn), Routledge, New York, USA, pp. 1-416.

46. Steenkamp J, Baumgartner H (1998) Assessing measurement invariance in cross-national consumer research. JCR 25(1): 78-90.

47. Byrne BM, Shavelson RJ, Muthen B (1989) Testing for the equivalence of factor covariance and mean structures: The issue of partial measurement invariance. Psychological Bulletin 105(3): 456-466.

48. Cheung GW, Rensvold RB (2002) Evaluating goodness-of-fit indexes for testing measurement invariance. Structural Equation Modeling 9(2): 233-255.

49. Wu AD, Li Z, Zumbo BD (2007) Decoding the meaning of factorial invariance and updating the practice of multi-group confirmatory factor analysis: A demonstration with TIMSS data. Practical Assessment Research and Evaluation 12(3): 1-26.

50. Hancock GR (1997) Structural equation modeling methods of hypothesis testing of latent variable means. Measurement and Evaluation in Counseling and Development 30: 91-105. 
51. Thompson MS, Green SB (2006) Evaluating between-group differences in latent variable means. In: GR Hancock \& RO Mueller (Eds.), A second course in structural equation modeling. Greenwich, Information Age, UK, pp. 119-169.

52. DeCarlo L (1997) On the meaning and use of kurtosis. Psychological Methods 2(3): 292-307.

53. Finney SJ, DiStefano C (2006) Nonnormal and categorical data in structural equation models. In GR Hancock \& RO Mueller (Eds.), A second course in structural equation modeling. Information Age, UK, pp. 269-314.

54. Kline R (1998) Principles and practice of structural equation modeling. Guilford Press, New York, USA, pp. 1-534.

55. Arbuckle JL (2006) AMOS (Version 7.0). SPSS, USA.
56. Fukunaka K (2007) Latent structured mean analysis for multi-group. In: H Toyoda (Ed.), Covariance structure analysis: AMOS. TokyoTosho, Japan pp. 67-99.

57. Cohen J (1988) Statistical power analysis for the behavioral sciences ( $2^{\text {nd }}$ edn), NJ Lawrence Erlbaum Associates, Publishers, USA.

58. Zimmerman BJ (1998) Developing self-fulfilling cycles of academic regulation: An analysis of exemplary instructional models. In: DH Schunk \& BJ Zimmerman (Eds.), Self-regulated learning: From teaching to self-reflective practice. Guilford, New York, USA, p. 1-19.

59. Meredith W (1993) Measurement invariance, factor analysis, and factorial invariance. Psychometrika 58(4): 525-543.

60. Little TD (1997) Mean and covariance structures (MACS) analysis of cross-cultural data: Practical and theoretical issues. Multivariate Behavioral Research 32(1): 53-76. 